\title{
Non-causal Digital Cancellation in Simultaneous Transmission and Reception Technology
}

\author{
Sun Bing ${ }^{1, *}$, Ruan Huai-lin ${ }^{1}$, and Zhong Hua $i^{1}$ \\ ${ }^{1}$ National University of Defense Technology, Hefei, China
}

\begin{abstract}
The electronic system occasionally requires the simultaneous transmission and reception of signals in the same platform. In the circumstance of simultaneous transmission and reception, the strong coupling interference signal cancellation requires shorter data and better cancellation effectiveness, but the cancellation performance of general causal canceller is relatively poor. Motivated by this, adopting non-causal filter to realize digital cancellation is then proposed in the paper. The model of non-causal filter digital cancellation is built at first, and the digital cancellation is simulated and analysed. The simulation results demonstrate that the digital cancellation has outstanding characteristics, and at the same time, the results also indicate that in the circumstance of the same filter order, the non-causal filter digital cancellation has better cancellation effectiveness.
\end{abstract}

\section{Introduction}

With the development of electronic countermeasure and radar, the demand of electronic countermeasure system has grown constantly. In many cases, in order to promote the viability and combat performance of electronic jammers, the jammers need to reconnoiter the surrounding radar equipment continuously, and launch jamming signals to the enemy radars uninterruptedly. Therefore, the jammers require the simultaneous transmission and reception [1, 2]. When the system works at the mode of simultaneous transmission and reception, the system often adopts some measures of transceiver isolation $[3,4]$ to ensure that the interference signals coupled to the receiver does not influence the normal work of the receiver. Digital cancellation [5] is a common isolation technology, which can improve the output SNR of receiver, as well as promote the transceiver isolation ratio.

When the system works at the mode of simultaneous transmission and reception, the reconnaissance receiving antennas receive radar signals from the target radars and jamming signals from the interference transmitting antennas at the same time. The effective reconnaissance for enemy radar signals requires the cancelation of the interference signals. Recently, LMS [6] algorithm and variable step LMS algorithm are often used in the adaptive filtering. In theory, this kinds of algorithm can filter the coupling interference signals when the filter order is long enough, but in practical application, the real-time require shorter order. And the coupling interference signals transform from digital interference signals to analog interference signals through D/A conversion, then enter the receiver after the space coupling delay, and then transform into digital signals again through $\mathrm{A} / \mathrm{D}$ conversion for processing. In the process of digital cancellation, the mathematical model is more tally with the non-causal relationship, therefore, adopting non-causal filter for digital cancellation is more tally with the actual situation. The interference signal data stored in the digital memory can be directly called as a reference signal due to the jamming signals that transmitted by our own, which is used to eliminate the interference signals coupled to reconnaissance receiver, thus It is feasible to replace causal system with non-causal filter system. In this case, this paper proposes that we can adopt the non-causal digital cancellation system, which can achieve better cancellation effectiveness compared to causal system, as well as improve digital cancellation effectiveness.

\section{Digital cancellation model}

The functional block diagram of adaptive digital cancellation is as shown in figure 1 . The received signals transform into digital signals through $\mathrm{A} / \mathrm{D}$ conversion:

$$
x(n)=s(n)+J^{\prime}(n)
$$

\footnotetext{
* Corresponding author: 13721052122@163.
} 
where $s(n)=s_{a}(n T)$ is the useful radar signal, $J^{\prime}(n)=J_{a}^{\prime}(n T)$ is the coupling interference signal, $T$ is the sampling interval. Reference signal $J(n)$ is the jamming signal that called from digital storage directly, which has correlation with the coupling interference signal $J^{\prime}(n)$.

The correlation lies in: the jamming decision generate digital jamming signal $J(n), J(n)$ transform through D/A conversion to generate analog signal $J_{a}(t)$, and $J_{a}(t)$ will be transmitted by interference transmitting antenna after up-conversion. In the launching process, $J_{a}(t)$ enter into the reconnaissance receiving antenna through space coupling channel. The coupling interference signal $J_{a}^{\prime}(t)$ received by reconnaissance receiving antenna is generated by $J_{a}(t)$ after attenuation and delay, then digital coupling interference signal $J^{\prime}(n)$ will be gained through sampling.

In the circumstance of the relatively weak correlation between received radar signal and coupling interference signal, the adaptive filter will automatically modulate its own parameters to make the output $y(n)$ become the best estimated value of $J^{\prime}(n)$ 。At this moment, the error signal $e(n)$ in figure 1 will approach radar signal $s(n)$, which can eliminate the coupling interference signal and improve the SNR.

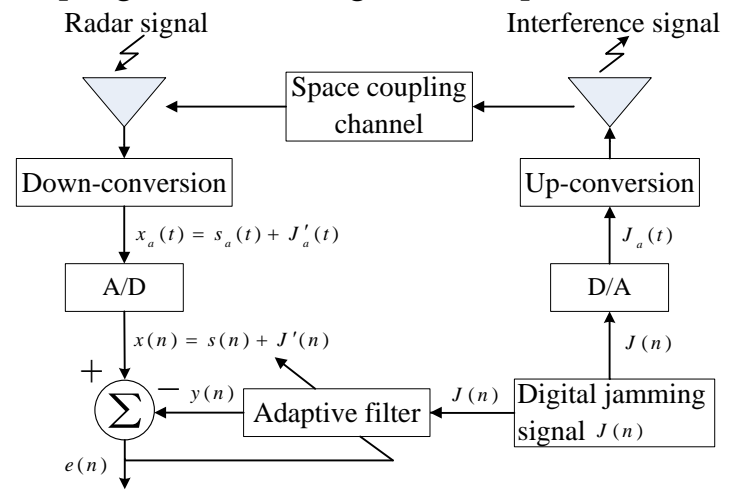

Fig. 1. Model of adaptive digital cancellation

\section{Non-causal cancellation cancellation}

Jamming signal $J_{a}(t)$ is generally gained from the jamming modulated digital signal $J(n)$ after passing through D/A conversion and low-pass filtering, the jamming signal $J_{a}(t)$ will be then transmitted by antenna after up-conversion and amplification. If you ignore the near field coupling effect and multipath transmission coupling, the digital jamming signal $J(n)$ and $J_{a}(t)$ can be approximately expressed as follows:

$$
\begin{aligned}
J_{a}(t) & =\sum_{n=-\infty}^{\infty} J(n) \cdot h_{n}(t-\delta) \\
& =\sum_{n=-\infty}^{\infty} J(n) \cdot \frac{\sin [\pi(t-\delta-n T) / T]}{\pi(t-\delta-n T) / T}
\end{aligned}
$$

where $T$ is the sampling interval, $h_{n}(t)$ is a spline function, the spline function is $\sin c$ function, where the $\delta$ means the conversion time error. Coupling jamming signal $J_{a}(t)$ is gained from the coupling analog jamming signal $J_{a}^{\prime}(t)$ after A/D conversion:

$$
J^{\prime}(n)=J_{a}^{\prime}(n T)
$$

The coupling analog jamming signal $J_{a}^{\prime}(t)$ is gained from the jamming signals after the attenuation and delay. Here we take the narrow-band signal for example:

$$
J_{a}^{\prime}(t)=\alpha J_{a}(t-\tau)
$$

where $\alpha$ is attenuation factor, $\tau$ is delay time.

To make digital cancellation for the coupling jamming signal, the fundamental demand is the exact estimation for the jamming signal $J^{\prime}(n)$, and the jamming signal $J^{\prime}(n)$ is then removed from the received signals. By substituting (4) into (3), we can rewrite (3) as

$$
J^{\prime}(n)=J_{a}^{\prime}(n T)=\alpha J_{a}(n T-\tau)
$$

Then we substitute (2) into (5) and rewrite (5) as

$$
J^{\prime}(n)=\alpha \cdot \sum_{m=-\infty}^{\infty} J(m) \cdot \frac{\sin [\pi(n T-\tau-\delta-m T) / T]}{\pi(n T-\tau-\delta-m T) / T}
$$

The equation above is the relational expression between the reference jamming digital signal $J(n)$ and the coupling digital signal $J^{\prime}(n)$. According to the delay time $\tau+\delta$, the question can be divided into two cases:

(1)When the $\tau+\delta$ is integer times of sampling interval, namely the delay time $\tau+\delta=p T, p=0,1,2,3 \cdots$, (6)can be rewritten as

$$
J^{\prime}(n)=\alpha J[(n-p)]
$$

At this time only a sample point of digital storage jamming signal $J[(n-p)]$ is needed to eliminate $J^{\prime}(n)$, however, in general, $\tau+\delta$ isn't integer times of sampling interval.

(2)When $\tau+\delta$ isn't integer times of sampling interval, namely $\tau+\delta \neq p T, p=0,1,2,3 \cdots$ 。 So we can assume the delay time $\tau+\delta=p T+\beta, 0<\beta<T$, $k=0,1,2,3 \cdots$ and (6) can be rewritten as

$$
J^{\prime}(n)=\sum_{k=-\infty}^{\infty} w_{k} J(n-k)
$$

where $w_{k}$ is weight coefficient as 


$$
w_{k}=\alpha \cdot \frac{\sin [\pi(k-p)-\pi \cdot \beta / T]}{\pi(k-p)-\pi \cdot \beta / T}
$$

From (8), we can acquire that in ideal situation an infinite number of sample points of reference signal are needed to eliminate the coupling jamming signal $J^{\prime}(n)$. However we can only select limited sample points of reference signal to realize the cancellation of the coupling interference signal $J^{\prime}(n)$, so the selection of sample points is very important. This limited length of causal cancellation and limited length of non-causal cancellation are mainly discussed and analyzed in the paper. Let us assume that the filter has $N$ orders as shown in figure 2 .

(1) For the cancellation system that adopted the causal filter, we can use the $N$ sample points before $(n-p) T$ of the reference signal $J(n)$ to estimate $J^{\prime}(n)$, namely as

$$
J^{\prime}(n)=\sum_{k=p}^{p+N-1} w_{k} J(n-k)
$$

if the delay time $\tau+\delta<T, p=0$, thus (10) can be rewritten as

$$
J^{\prime}(n)=\sum_{k=0}^{N-1} w_{k} J(n-k)
$$

This is as same as the expression of general causal filter system.

(2) For the cancellation system that adopted the non-causal filter, we can adopt the $N_{1}$ sample points and the subsequently $N-N_{1}$ sample points of reference signal $J(n)$ to estimate $J^{\prime}(n)$, namely as

$$
J^{\prime}(n)=\sum_{k=p-N+N_{1}}^{p+N_{1}-1} w_{k} J(n-k)
$$

Reference signal

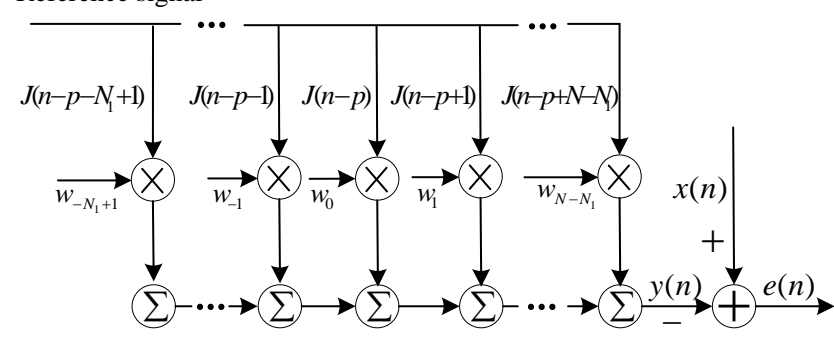

where $0<N_{1}<N$.

Fig. 2. Digital canceler

\section{Simulation and results}

The causal digital cancellation and non-causal digital cancellation are simulated and analyzed as follows.

The variable step-size least mean-square (VSS-LMS) algorithm is adopted in this paper, which can be summarized into the following three parts:
1) the filter output:

$$
y(n)=\boldsymbol{w}^{T}(n) \boldsymbol{J}(n)
$$

2)the estimation error:

$$
e(n)=x(n)-y(n)
$$

3)the weight adaptive updating:

$$
\boldsymbol{w}(n+1)=\boldsymbol{w}(n)+\mu(n) e(n) \boldsymbol{J}(n)
$$

where $\boldsymbol{w}(n)$ is adaptive filter weight vector in the $n$ time, $\mu(n)$ is the iteration step size parameter as

$$
\mu(n)=\beta\left(\frac{1}{1+\exp \left(-\alpha|e(n)|^{m}\right)}-0.5\right)
$$

where $\alpha$ is a constant to control the shape of the function, $\beta$ is a constant to control the value range of the function, $m$ is a constant. The parameter settings of our VSS-LMS are $\alpha=3000, \alpha=3000, m=3$ 。 Notice that at each moment, the product of the input vector that is defined by the step length and the error of estimation is served as the adjustment of the weight coefficient.

Assuming that target radar signal is linear frequency modulation signal, amplitude is $1 \mathrm{~V}$. Jamming signal is continuous emission of noise frequency modulation signal, frequency modulation slope is $2 \times 10^{7} \mathrm{MHz} / \mathrm{s}$, signal amplitude is $1 \mathrm{~V}$, sampling interval is $2 n s$ as well as $\tau+\delta=0.2 T$. The filter order is eight-order, and step length $\mu$ is 0.01 . The process and results of digital cancellation are shown in figure 3.

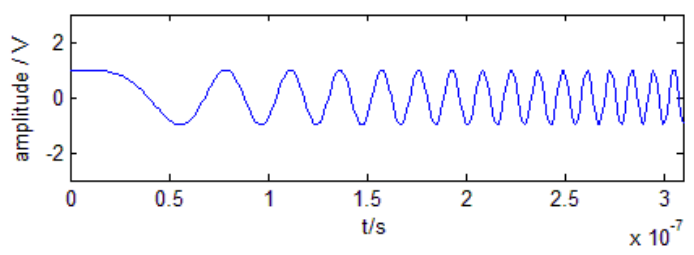

(a)Target radar signal time domain waveform

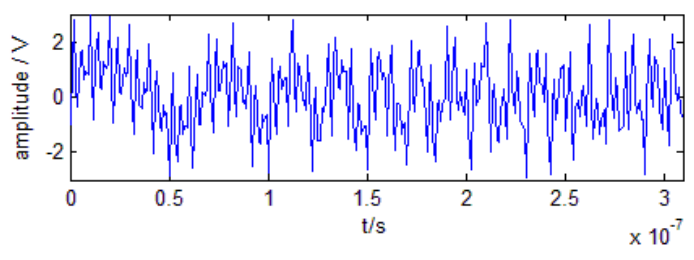

(b)Time domain waveform of practical receiving signal for receiving antenna 

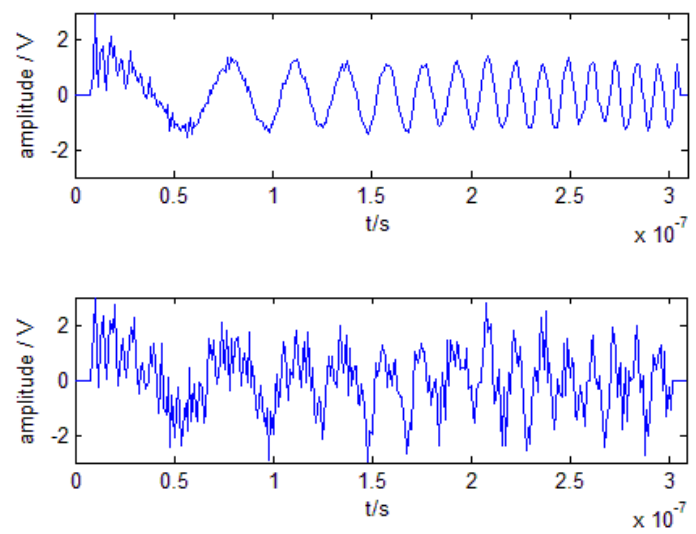

(c)Output signal time domain waveform of non-causal digital cancellation and causal digital cancellation
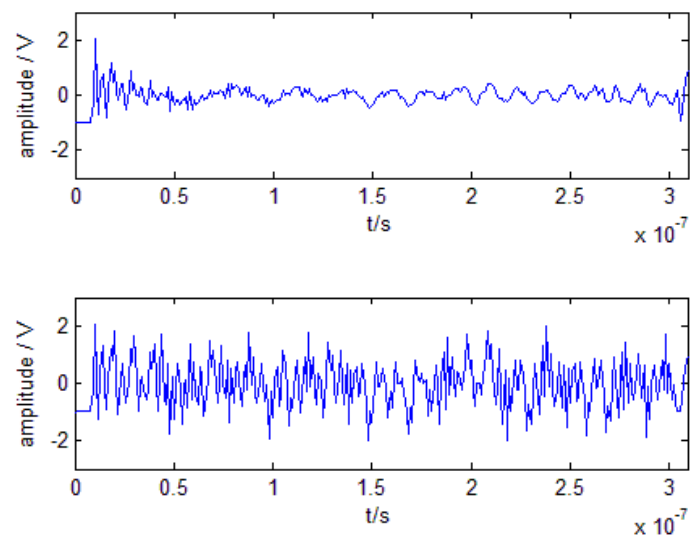

(d)Absolute error between cancellation output signal and radar signal of non-causal digital cancellation and causal digital cancellation

Fig.3. Digital cancellation simulation

In figure 3, (a) is the time domain waveform of target radar signals in the reconnaissance receiving antenna. (b) is the time domain waveform of practical receiving signals in reconnaissance receiving antenna. The practical receiving signals are actually the mixture signals of the target radar signals and the coupling interference signals, and due to the fact that the intensity of coupling interference signals is greater than the intensity of target radar signal, the radar signals are completely submerged in the coupling interference signal, and the target radar signals can't be detected and analyzed by the reconnaissance receiver. (c) is the output signal time domain waveform of non-causal digital cancellation and causal digital cancellation. It can be seen that the output signal time domain waveform after the cancellation is consistent with the received radar signal time domain waveform, the coupling interference signal whose power greater than the radar signal has been basically eliminated after digital cancellation. Compared to the time domain waveform after causal cancellation, the time domain waveform after the non-cause digital cancellation is closer to the desired target radar signal. (d) is the absolute error between the output signals and the radar signals, the output signals are from the non-causal cancellation and causal cancellation. It can be seen that the output result of the non-causal digital cancellation has smaller error and better cancellation effect compared to the output result of the causal digital cancellation.

\section{Conclusion}

In the circumstance of the same filter order, the VSSLMS algorithm with the same parameter settings is adopted by both the causal cancellation system and the non-causal cancellation system to compare their performance. Therefore, the computation results are same. If the cancellation effects of causal cancellation and non-cancel cancellation are wanted to be same, the causal order cancellation system requires higher filter order, which will has greater amount of computation compared to the non-causal cancellation system.

\section{References}

1. Ahmedand, E., Eltawil, A.M.: 'All-digital selfinterference cancellation technique for full-duplex systems wireless communications, IEEE Transactions on Wireless Communications, 2015, 14, (7), pp. 3519-3532, DOI: 10.1109/TWC.2015.240787

2. Sahai, A., Patel G., Dick C.: 'On the impact of phase noise on active cancelation in wireless fullduplex', IEEE Transactions on Vehicular Technol, 2013, 62, (9), pp. 4494-4510, DOI: 10.1109/TVT.2013.2266359

3. Cooper, K.B., Dengler, R.J.: 'Residual radar in a submillimeter-wave FMCW isolation phase noise and transmit/receive', IEEE MTT-S International Conference on Microwave Symposium, Tampa, 2014, pp. 1-4, DOI: 10.1109/MWSYM.2014.6848268

4. Krishnamoorthy, G., Ashok, P., Tesar, D.: 'Simultaneous Sensor and Process Fault Detection and Isolation in Multiple-Input-Multiple-Output Systems', IEEE Systems Journal, 2015, 9, (2), pp. 335 - 349, DOI: 10.1109/JSYST.2014.2307632

5. LI, J., Zhang, H., Fan, M.: 'Digital Self-Interference Cancellation Based on Independent Component Analysis for Co-Time Co-frequency Full-Duplex Communication Systems', IEEE Access, 2017, 5,pp.1022210231,DOI:10.1109/ACCESS.2017.2712614

6. Chien, Y.R., Tseng, W.J.: 'Switching-based variable step-size approach for partial update LMS algorithms, Electronics Letters, 2013, 49,(17),pp.1081-1083,DOI:10.1049/el.2013.1762 